\title{
Down-regulation of TRPV6 Is Associated With Adverse Prognosis in Hepatocellular Carcinoma Treated With Curative Resection
}

\author{
HYUN HEE KOH ${ }^{1}$, SANGJOON CHOI ${ }^{1}, \mathrm{CHEOL}-K E U N ~ P A R K^{1,2}$ and SANG YUN HA ${ }^{1}$ \\ ${ }^{1}$ Department of Pathology and Translational Genomics, Samsung Medical Center, \\ Sungkyunkwan University School of Medicine, Seoul, Republic of Korea; \\ ${ }^{2}$ Anatomic Pathology Reference Lab, Seegene Medical Foundation, Seoul, Republic of Korea
}

\begin{abstract}
Background/Aim: Transient receptor potential vanilloid 6 (TRPV6), an endothelial $\mathrm{Ca}^{2+}$-selective entry channel, is expressed in various cancer types, and a selective TRPV6 inhibitor is currently being investigated in a clinical trial. However, TRPV6 expression in hepatocellular carcinoma (HCC) has not been reported. Materials and Methods: We evaluated TRPV6 expression in 219 cases of HCC and analyzed its association with clinicopathological parameters and prognostic significance. TRPV6 mRNA expression was compared between HCC and non-tumor liver tissues using various public datasets, and its prognostic effect was examined in The Cancer Genome Atlas (TCGA) cohort. Results: Low TRPV6 expression was found in $37.4 \%$ of patients, which was significantly associated with adverse histologic features, and patients with low TRPV6 expression had shorter recurrence-free and disease-free survival. TRPV6 mRNA expression was consistently lower in HCC compared to non-tumor liver samples in public datasets, at the whole tissue level as well as single-cell level. Patients with low TRPV6 expression in the TCGA cohort had shorter progression-free survival. Conclusion: TRPV6 expression is down-regulated in HCCs and associated with a poor prognosis. TRPV6 may be a prognostic biomarker in $\mathrm{HCC}$.
\end{abstract}

This article is freely accessible online.

Correspondence to: Sang Yun Ha, MD, Ph.D., Department of Pathology and Translational Genomics, Samsung Medical Center, Sungkyunkwan University School of Medicine, 81 Irwon-ro, Gangnam-gu, Seoul 06351, Republic of Korea. Tel: +82 234101586, Fax: +82 234100025, e-mail: sangyun.ha@skku.edu

Key Words: Hepatocellular carcinoma, prognosis, recurrence, TRPV6, biomarkers.
Hepatocellular carcinoma (HCC) is one of the most common cancers and a leading cause of cancer-related deaths worldwide (1). Although surgical resection is the treatment of choice for HCC, the prognosis of HCC patients after surgery generally tends to be poor due to the high frequency of tumor recurrence (2). Sorafenib chemotherapy has been used, until now, as the most effective systemic treatment for advanced HCC, and there is still a continuous search for a new agent for treating advanced HCC. Novel targeted agents, such as regorafenib and lenvatinib, and immune checkpoint inhibitors, such as nivolumab and pembrolizumab, have been approved by the US Food and Drug Administration (3-6). However, owing to the limitation of applying these therapies $(4,6)$, further therapeutic agents need to be developed and reliable molecular biomarkers for predicting the response of those agents and prognosis ought to be discovered in the era of precision medicine (7).

Transient receptor potential vanilloid 6 (TRPV6), the major constituent of TRP channels, is an epithelial calcium channel located in the epithelium of organs including the digestive tract, testis, placenta, and kidney with highly selective affinity to divalent cations, particularly $\mathrm{Ca}^{2+}(8)$. Owing to its high selectivity to $\mathrm{Ca}^{2+}$, it plays a critical role in $\mathrm{Ca}^{2+}$ homeostasis in physiological conditions $(8,9)$. Also, it is clarified that $\mathrm{Ca}^{2+}$ signaling is central to the major cancer hallmark processes, including uncontrolled cell proliferation, resistance to apoptosis, tissue invasion, and angiogenesis $(10,11)$. Thus, it is expected that TRPV6 functions as an oncogene in carcinogenesis. TRPV6 expression is variable and depends on the organ and cancer type (8), but little is known about the exact role and mechanism through which TRPV6 contributes to carcinogenesis. In recent days, accumulating evidence indicates that TRPV6 expression, either over-expression or down-regulation, is related to cancer progression. Overexpression of TRPV6 is observed in prostate, breast, and thyroid cancers $(8,12,13)$, whereas down-regulation of TRPV6 is observed in esophageal, non-small cell lung, and 
renal cancers (14-16). Also, a selective TRPV6 inhibitor has been developed, and its pharmacological effect and safety were recently shown in humans (17). However, the association between the TRPV6 expression and clinicopathological outcome in patients with HCC has not been studied yet.

In this study, we evaluated TRPV6 expression in surgically resected HCC samples using immunohistochemistry (IHC) and analyzed its association with clinicopathological parameters and prognostic effect. Then, we aimed to elucidate the possibility of using TRPV6 as a promising prognostic predictor in patients with HCC.

\section{Materials and Methods}

Patients and specimens. Initially, a total of 283 patients treated with curative hepatectomy as the first line of treatment for primary HCC between July 2000 and May 2006 at the Samsung Medical Center, Seoul, Republic of Korea were enrolled in this study. Sixty-four patients who presented with insufficient tissue for tissue microarray (TMA) were excluded, and finally 219 patients were included in the study cohort.

Curatively resected tumors had clear resection margins confirmed microscopically and no residual tumors confirmed by radiological examination one month after surgery. Tumor staging was performed according to both the American Joint Committee on Cancer (AJCC) staging system ( $8^{\text {th }}$ edition) (18) and Barcelona Clinic Liver Cancer (BCLC) staging classification (19). Intrahepatic metastasis and multicentric occurrence were defined according to previously defined criteria (20), and tumor necrosis or tumor-infiltrating lymphocytes (TILs) were determined as described previously (21). All patients were followed up every 3 months after surgery, with three-phase dynamic computed tomography scans or magnetic resonance imaging and serum $\alpha$ fetoprotein (AFP) level evaluations. Recurrence-free survival (RFS) and disease-specific survival (DSS) were defined as the difference between the date of surgery and date of recurrence or HCC-related death, respectively (22). The Institutional Review Board of the Samsung Medical Center approved this study and waived the need for informed consent (IRB No. 2021-05-036).

$I H C$. IHC was performed using TMA consisting of two $2 \mathrm{~mm}$ cores of HCC tissue as previously described (23). The sections were incubated with a rabbit anti-TRPV6 antibody (ACC-036, Alomone labs, Jerusalem, Israel) for $60 \mathrm{~min}$ at room temperature, after antigen retrieval with LOW buffer $(\mathrm{pH}$ 6.0) in DAKO PT Link (Agilent, Glostrup, Denmark) at $97^{\circ} \mathrm{C}$ and blocking with Agilent Protein Block, Blocking x0909 (Agilent) for $15 \mathrm{~min}$. Antigen-antibody chromogenic reactions were developed using the Agilent EnVision FLEX Systems K8002 (Agilent) for $30 \mathrm{~min}$. Prostate adenocarcinoma and normal prostate tissues were used as positive and negative controls, respectively, according to the manufacturer's recommendations. Cytoplasmic staining of TRPV6 in tumor cells was regarded as positive, and TRPV6 staining was analyzed by a semi-quantitative method using $\mathrm{H}$-score, as described previously (23). Briefly, $\mathrm{H}$-score was generated on a continuous scale of 0 to 300 by multiplying the percentage of stained cells $(0-100 \%)$ and 4 intensity categories $(0$ for negative, $1+$ for weak, 2+ for moderate, and 3+ for strong positive) (Figure 1A-D).

Analysis of gene expression omnibus (GEO) databases and The Cancer genome atlas (TCGA). The TRPV6 expression data in microarray datasets were downloaded from the National Center for Biotechnology Information (NCBI) GEO database, specifically the GSE14520 (24) series on the GPL571 platform (Affymetrix GeneChip Human Genome U133A 2.0 Array, Santa Clara, CA, USA), GSE54236 (25) series on the GPL6480 platform (Agilent-014850 Whole Human Genome Microarray 4×44K G4112F, Santa Clara, CA, USA), and GSE89377 (26) series on the GPL6947 platform (Illumina HumanHT12 V3.0 expression BeadChip, San Diego, CA, USA). The mRNA microarray datasets from 22, 81, and $40 \mathrm{HCC}$ tissue samples and 21, 80, and 67 normal liver tissue samples were obtained from GSE14520, GSE54236, and GSE89377, respectively. In particular, GSE89377 contained various liver tissues classified as nontumor, including chronic hepatitis and cirrhosis $(n=45)$, lowgrade dysplastic nodules $(n=11)$, high-grade dysplastic nodules $(n=11)$, early HCC $(n=5)$, and HCC grade I-III $(\mathrm{n}=35)$. The TRPV6 expression data of the single-cell RNAseq dataset, GSE149614 (27) series on the GPL24676 platform [Illumina NovaSeq 6000 (Homo sapiens)], was also downloaded from the NCBI GEO database. GSE149614 contains a total of 21 samples of the primary tumor $(n=10)$, portal vein tumor thrombus $(\mathrm{n}=2)$, metastatic lymph node $(\mathrm{n}=1)$, and non-tumor liver samples $(\mathrm{n}=8)$ from 71,915 cells of 10 patients with HCC. The normalized data of 20 samples of primary tumor, portal vein tumor thrombus, and nontumor liver with a total of 69,072 cells was downloaded and analyzed in this study. TRPV6 expression was compared between normal hepatocytes and HCC tumor cells, after summing the expression of single-cell RNAseq values for each sample. Probes were converted into corresponding gene symbols according to the annotation information of each platform. In addition, the TRPV6 mRNA expression data and relevant clinical data of 424 samples from the Liver Hepatocellular Carcinoma (LIHC) TCGA cohort were downloaded from the TCGA LIHC RNAseq dataset using the Genomic Data Commons Data Portal in October 2021 (28). The LIHC TCGA cohort contained 371 primary tumor samples, 3 recurrent tumor samples, and 50 normal samples; survival data were provided for 366 out of 371 primary tumor samples. 


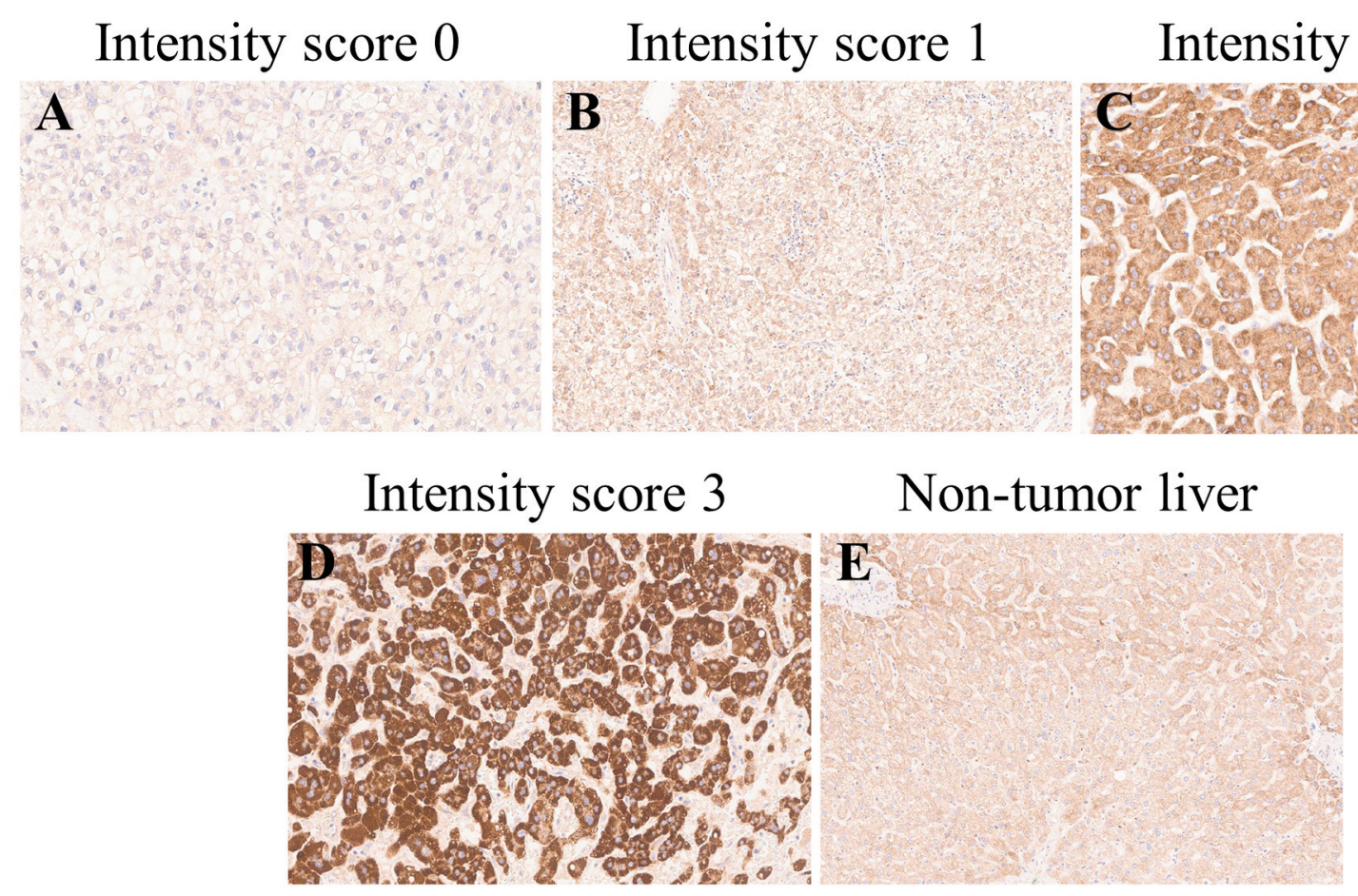

Figure 1. Representative images of TRPV6 immunohistochemistry according to intensity score calculated using the H-score system.

Statistical analysis. We used the X-tile statistics software (Yale University, New Haven, CT, USA) to determine the optimal cut-off value of TRPV6 protein and mRNA expression with the most significant difference in RFS or DFS (29). To analyze the relationship between TRPV6 expression and clinicopathological parameters, Pearson's chi square, Fisher's exact, or CochranArmitage test was used as appropriate. Kaplan-Meier survival curve was used to analyze survival rates, which were compared using the log-rank test. Cox's proportional hazard regression model was used for assessing the association between clinicopathological factors and survival time. Prognostic factors with $p$-values less than 0.05 in the univariable analysis were included in a multivariable analysis. Mann-Whitney $U$-test was used for comparing the mRNA expression between non-tumor tissue and HCC tissue. We examined the proportional hazard assumption graphically. Two-sided $p$-values less than 0.05 were considered statistically significant. Statistical analyses were performed using IBM SPSS for Windows (IBM Corp., Armonk, NY, USA) and R 4.1.1 (R Foundation for Statistical Computing, Vienna, Austria).

\section{Results}

Clinicopathological characteristics. Clinicopathological characteristics of patients are presented in Table I. Among the 219 HCC cases, there were 181 male patients $(82.6 \%)$ and 38 female patients (17.4\%), with ages ranging from 17 to 74 years old (mean: 52 years) and size of tumors ranging from 10 to $210 \mathrm{~mm}$ (mean: $51 \mathrm{~mm}$ ). By preoperative examination, 195 patients $(89.0 \%)$ were hepatitis virus carriers, of which the majority $(174 ; 79.5 \%)$ had hepatitis B virus followed by hepatitis $\mathrm{C}$ virus $(19 ; 8.7 \%)$. Twenty-six patients $(11.9 \%)$ were hypo-albuminemic (serum albumin level $\leq 3.5 \mathrm{~g} / \mathrm{dl}$ ), and 82 patients $(37.4 \%)$ showed increased AFP levels (>200 ng/ml). By microscopic examination, 122 $(55.7 \%), 8(3.7 \%)$, and $54(24.7 \%)$ patients displayed microvascular invasion, major portal vein invasion, and intrahepatic metastasis, respectively. In addition, 54 cases (24.7\%) displayed tumor necrosis, and 66 cases $(30.1 \%)$ showed moderate to high numbers of TILs. The majority $(177 ; 80.8 \%)$ of cases were Edmonson grade II, followed by grade I $(24 ; 11.0 \%)$ and grade III $(18 ; 8.2 \%)$. Regarding nontumor background liver, 114 cases $(52.1 \%)$ showed cirrhosis. Based on the T staging of AJCC, there were 90 (41.1\%), 90 $(41.1 \%), 34(15.5 \%)$, and $5(2.3 \%)$ patients with I, II, III, and IV stage, respectively. According to follow-up after surgery, 145 patients $(66.2 \%)$ recurred, and their RFS ranged from 0.33 to 149.5 months.

TRPV6 expression in HCC tissues and its association with clinicopathological features. TRPV6 was expressed in the cytoplasm of HCC tumor cells (Figure 1A-D). The median 
Table I. Association between TRPV6 expression and clinicopathological variables.

\begin{tabular}{|c|c|c|c|c|}
\hline \multirow[t]{2}{*}{ Variables } & \multirow[t]{2}{*}{ Total } & \multicolumn{2}{|c|}{ TRPV6 IHC H-score } & \multirow[b]{2}{*}{$p$-Value } \\
\hline & & $<100(\mathrm{n}=82)$ & $\geq 100(\mathrm{n}=137)$ & \\
\hline Gender & & & & 0.307 \\
\hline Female & 38 & $17(44.7 \%)$ & $21(55.3 \%)$ & \\
\hline Male & 181 & $65(35.9 \%)$ & $116(64.1 \%)$ & \\
\hline Age & & & & 0.067 \\
\hline$<40$ & 23 & $13(56.5 \%)$ & $10(43.5 \%)$ & \\
\hline$\geq 40$ & 196 & $69(35.2 \%)$ & $127(64.8 \%)$ & \\
\hline Tumor size & & & & 0.014 \\
\hline$<5 \mathrm{~cm}$ & 130 & $40(30.8 \%)$ & $90(69.2 \%)$ & \\
\hline$\geq 5 \mathrm{~cm}$ & 89 & $42(47.2 \%)$ & $47(52.8 \%)$ & \\
\hline Edmondson grade & & & & 0.016 \\
\hline I & 24 & $6(25.0 \%)$ & $18(75.0 \%)$ & \\
\hline II & 177 & $64(36.2 \%)$ & $113(63.8 \%)$ & \\
\hline III & 18 & $12(66.7 \%)$ & $6(33.3 \%)$ & \\
\hline Microvascular invasion & & & & $<0.001$ \\
\hline$(-)$ & 97 & $21(21.6 \%)$ & $76(78.4 \%)$ & \\
\hline$(+)$ & 122 & $61(50.0 \%)$ & $61(50.0 \%)$ & \\
\hline Major portal vein invasion & & & & $<0.001$ \\
\hline$(-)$ & 211 & $74(35.1 \%)$ & $137(64.9 \%)$ & \\
\hline$(+)$ & 8 & $8(100.0 \%)$ & $0(0.0 \%)$ & \\
\hline Intrahepatic metastasis & & & & 0.002 \\
\hline$(-)$ & 165 & $52(31.5 \%)$ & $113(68.5 \%)$ & \\
\hline$(+)$ & 54 & $30(55.6 \%)$ & $24(44.4 \%)$ & \\
\hline Multicentric occurrence & & & & 0.938 \\
\hline$(-)$ & 206 & $77(37.4 \%)$ & $129(62.6 \%)$ & \\
\hline$(+)$ & 13 & $5(38.5 \%)$ & $8(61.5 \%)$ & \\
\hline AJCC T stage $8^{\text {th }}$ & & & & $<0.001$ \\
\hline 1 & 90 & $19(21.1 \%)$ & $71(78.9 \%)$ & \\
\hline 2 & 90 & $41(45.6 \%)$ & $49(54.4 \%)$ & \\
\hline 3 & 34 & $21(61.8 \%)$ & $13(38.2 \%)$ & \\
\hline 4 & 5 & $1(20.0 \%)$ & $4(80.0 \%)$ & \\
\hline BCLC stage & & & & 0.002 \\
\hline $0, \mathrm{~A}$ & 117 & $34(29.1 \%)$ & $83(70.9 \%)$ & \\
\hline B & 92 & $40(43.5 \%)$ & $52(56.5 \%)$ & \\
\hline $\mathrm{C}$ & 10 & $8(80.0 \%)$ & $2(20.0 \%)$ & \\
\hline Albumin level, g/dl & & & & 0.585 \\
\hline$>3.5$ & 193 & $71(36.8 \%)$ & $122(63.2 \%)$ & \\
\hline$\leq 3.5$ & 26 & $11(42.3 \%)$ & $15(57.7 \%)$ & \\
\hline AFP level, $\mathrm{ng} / \mathrm{ml}^{\mathrm{a}}$ & & & & 0.015 \\
\hline$\leq 200$ & 129 & $40(31.0 \%)$ & $89(69.0 \%)$ & \\
\hline$>200$ & 82 & $39(47.6 \%)$ & $43(52.4 \%)$ & \\
\hline Etiology & & & & 0.127 \\
\hline Non-viral & 24 & $10(41.7 \%)$ & $14(58.3 \%)$ & \\
\hline $\mathrm{HBV}$ & 174 & $69(39.7 \%)$ & $105(60.3 \%)$ & \\
\hline $\mathrm{HBV}$ and $\mathrm{HCV}$ & 2 & $0(0.0 \%)$ & $2(100.0 \%)$ & \\
\hline $\mathrm{HCV}$ & 19 & $3(15.8 \%)$ & $16(84.2 \%)$ & \\
\hline Liver cirrhosis & & & & 0.930 \\
\hline$(-)$ & 105 & $39(37.1 \%)$ & $66(62.9 \%)$ & \\
\hline$(+)$ & 114 & $43(37.7 \%)$ & $71(62.3 \%)$ & \\
\hline Tumor necrosis & & & & 0.002 \\
\hline$(-)$ & 165 & $52(31.5 \%)$ & $113(68.5 \%)$ & \\
\hline$(+)$ & 54 & $30(55.6 \%)$ & $24(44.4 \%)$ & \\
\hline Tumor infiltrating lymphocytes & & & & 0.930 \\
\hline$(-)$ & 153 & $57(37.3 \%)$ & $96(62.7 \%)$ & \\
\hline$(+)$ & 44 & $16(36.4 \%)$ & $28(63.6 \%)$ & \\
\hline$(++)$ & 22 & $9(40.9 \%)$ & $13(59.1 \%)$ & \\
\hline
\end{tabular}

Values are presented as number (\%); AJCC: American Joint Committee on Cancer; BCLC: Barcelona Clinic Liver Cancer; AFP: $\alpha$-fetoprotein; HBV: hepatitis B virus; HCV: hepatitis C virus. ${ }^{a} \mathrm{AFP}$ evaluation was not applicable in 8 cases. 

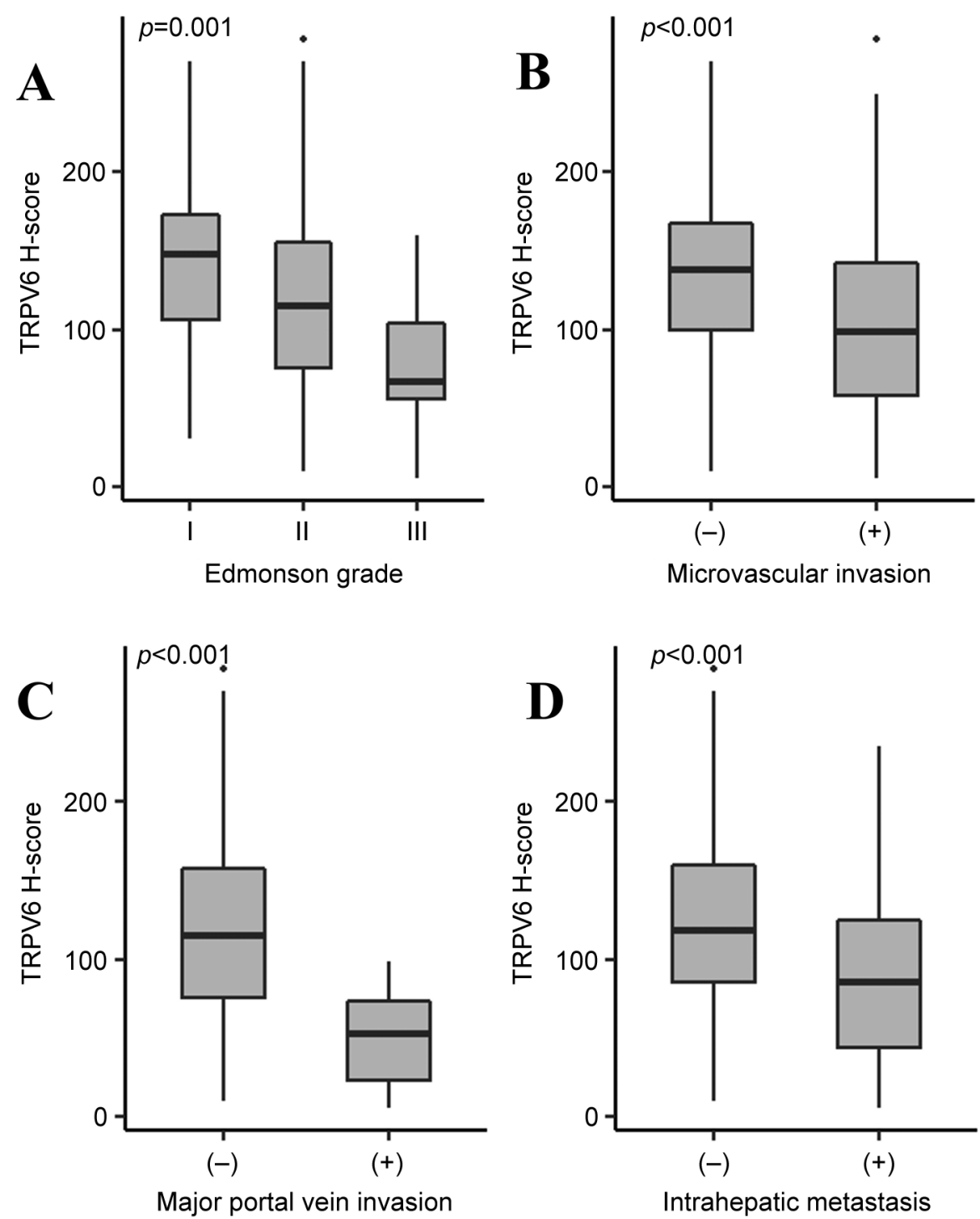

Figure 2. Boxplot comparing TRPV6 H-score according to clinicopathological variables. (A) Edmonson grade, (B) microvascular invasion, (C) major portal vein invasion, $(D)$ intrahepatic metastasis.

H-score of TRPV6 IHC was 112.5 (range=5-285) and the mean was 116.85 (standard deviation: 60.84). The non-tumor background liver tissue showed weak granular cytoplasmic staining (Figure 1E). The optimal cutoff value of TRPV6 expression in IHC analysis, determined using the X-Tile program with the highest statistical significance for RFS, was 100 . Among the 219 patients, $82(37.4 \%)$ were classified into the low expression group, while 137 (62.6\%) were classified into the high expression group. The association between TRPV6 expression and clinicopathological parameters is summarized in Table I. Low TRPV6 expression was more frequently found in patients with large tumor size $(p=0.014)$, intrahepatic metastasis $(p=0.002)$, and high serum AFP levels $(p=0.015)$. Furthermore, it was significantly associated with adverse histologic features known to predict adverse prognosis, including high Edmonson grade $(p=0.016)$, microvascular invasion $(p<0.001)$, and major portal vein invasion $(p<0.001)$ (Figure $2)$. The patients in the low expression group tended to have higher AJCC T or BCLC stage.

Impact of TRPV6 expression on the survival of patients with HCC. Patients with low TRPV6 expression showed significantly short RFS $(p=0.015)$ and DSS $(p=0.010)$ (Figure 3). In multivariable analysis, necrosis and intrahepatic metastasis were independent adverse 
Table II. Univariable and multivariable analysis for recurrence-free survival (RFS) and disease-specific survival (DSS).

\begin{tabular}{|c|c|c|c|c|c|c|c|}
\hline \multirow{2}{*}{$\begin{array}{l}\text { Univariable analysis } \\
\text { Variables }\end{array}$} & & \multicolumn{3}{|c|}{ RFS } & \multicolumn{3}{|c|}{ DSS } \\
\hline & & HR & $95 \% \mathrm{CI}$ & $p$-Value & HR & $95 \% \mathrm{CI}$ & $p$-Value \\
\hline Age & $<40$ years $v s . \geq 40$ years & 1.466 & $0.904-2.378$ & 0.121 & 1.598 & $0.845-3.025$ & 0.150 \\
\hline Tumor size & $>5 \mathrm{~cm} \mathrm{vs.} \leq 5 \mathrm{~cm}$ & 1.565 & $1.127-2.172$ & 0.007 & 2.887 & $1.833-4.548$ & $<0.001$ \\
\hline Edmonson grade & III $v s$. I+II & 1.851 & $1.083-3.166$ & 0.024 & 2.725 & $1.437-5.166$ & 0.002 \\
\hline Microvascular invasion & Yes vs. No & 2.316 & $1.645-3.259$ & $<0.001$ & 3.918 & $2.286-6.716$ & $<0.001$ \\
\hline Major portal vein invasion & Yes vs. No & 2.827 & $1.319-6.06$ & 0.008 & 5.732 & $2.613-12.574$ & $<0.001$ \\
\hline Intrahepatic metastasis & Yes vs. No & 4.666 & $3.254-6.69$ & $<0.001$ & 6.167 & $3.917-9.71$ & $<0.001$ \\
\hline Multicentric occurrence & Yes vs. No & 1.297 & $0.605-2.783$ & 0.504 & 0.896 & $0.328-2.452$ & 0.831 \\
\hline Albumin level, g/dl & $\leq 3.5 \mathrm{vs} .>3.5$ & 1.791 & $1.115-2.876$ & 0.016 & 2.109 & $1.159-3.837$ & 0.015 \\
\hline AFP level, ng/ml & $>200 v s . \leq 200$ & 1.861 & $1.333-2.598$ & $<0.001$ & 1.871 & $1.186-2.953$ & 0.007 \\
\hline Etiology & Viral vs. non-viral & 2.016 & $1.061-3.834$ & 0.032 & 1.986 & $0.803-4.916$ & 0.138 \\
\hline Liver cirrhosis & Yes $v s$. No & 1.400 & $1.007-1.947$ & 0.046 & 1.070 & $0.686-1.669$ & 0.765 \\
\hline Necrosis & Yes vs. No & 2.404 & $1.678-3.444$ & $<0.001$ & 4.176 & $2.664-6.546$ & $<0.001$ \\
\hline Tumor infiltrating lymphocytes & Yes vs. No & 0.712 & $0.493-1.029$ & 0.070 & 0.385 & $0.212-0.699$ & 0.002 \\
\hline TRPV6 IHC H-score & $\geq 100$ vs. $<100$ & 0.664 & $0.477-0.925$ & 0.015 & 0.559 & $0.358-0.874$ & 0.011 \\
\hline \multicolumn{2}{|l|}{ Multivariable analysis } & \multicolumn{3}{|c|}{ RFS } & \multicolumn{3}{|c|}{ DSS } \\
\hline Variables & & HR & $95 \% \mathrm{CI}$ & $p$-Value & HR & $95 \% \mathrm{CI}$ & $p$-Value \\
\hline Age & $<40$ years $v s . \geq 40$ years & & & & & & \\
\hline Tumor size & $>5 \mathrm{~cm} \mathrm{vs.} \leq 5 \mathrm{~cm}$ & 1.059 & $0.703-1.596$ & 0.784 & 1.525 & $0.904-2.573$ & 0.114 \\
\hline Edmonson grade & III $v s . \mathrm{I}+\mathrm{II}$ & 1.054 & $0.571-1.946$ & 0.866 & 1.310 & $0.627-2.736$ & 0.472 \\
\hline Microvascular invasion & Yes vs. No & 1.014 & $0.606-1.697$ & 0.958 & 0.799 & $0.357-1.789$ & 0.585 \\
\hline Major portal vein invasion & Yes vs. No & 0.586 & $0.242-1.42$ & 0.237 & 1.365 & $0.527-3.536$ & 0.522 \\
\hline Intrahepatic metastasis & Yes $v s$. No & 4.402 & $2.721-7.121$ & $<0.001$ & 6.307 & $3.309-12.022$ & $<0.001$ \\
\hline Multicentric occurrence & Yes vs. No & & & & & & \\
\hline Albumin level, g/dl & $\leq 3.5 v s .>3.5$ & 1.789 & $1.048-3.054$ & 0.033 & 1.634 & $0.798-3.346$ & 0.180 \\
\hline AFP level, ng/ml & $>200 v s . \leq 200$ & 1.370 & $0.958-1.961$ & 0.085 & 0.918 & $0.537-1.569$ & 0.754 \\
\hline Etiology & Viral vs. non-viral & 1.399 & $0.696-2.812$ & 0.346 & & & \\
\hline Liver cirrhosis & Yes vs. No & 1.418 & $0.979-2.054$ & 0.064 & & & \\
\hline Necrosis & Yes vs. No & 1.791 & $1.132-2.833$ & 0.013 & 3.212 & $1.781-5.793$ & $<0.001$ \\
\hline Tumor infiltrating lymphocytes & Yes vs. No & & & & 0.446 & $0.228-0.872$ & 0.018 \\
\hline TRPV6 IHC H-score & $\geq 100$ vs. $<100$ & 0.856 & $0.586-1.251$ & 0.422 & 0.890 & $0.528-1.5$ & 0.663 \\
\hline
\end{tabular}

IHC: Immunohistochemistry; AFP: $\alpha$-fetoprotein; HR: hazard ratio.

prognostic factors for RFS $(p=0.013$ and $<0.001$, respectively) and DSS ( $p=0.001$ and $<0.001$, respectively) (Table II). Low serum albumin level was observed to be an independent adverse prognostic factor for only RFS $(p=0.033)$. TRPV6 expression fails to show an independent prognostic effect.

TRPV6 mRNA expression in public data. We compared the expression of TRPV6 mRNA in non-tumor liver and HCC tissues from five public gene expression datasets GSE14520, GSE54236, GSE89377, GSE149614, and TCGA HCC cohorts. The mean TRPV6 mRNA expression in HCC tissue was significantly lower than that in nontumor liver tissue in all GEO datasets, not only at the whole tissue level (GSE14520, $p=0.006$; GSE89377, $p<0.001$;
GSE54236, $p<0.001$ ) (Figure 4A-C), but also at the individual cell level at single-cell sequencing dataset (GSE149614, $p=0.02$ ) (Figure 4D). Particularly, GSE89377 contained various liver tissues classified as non-tumor, lowgrade dysplastic nodule, high-grade dysplastic nodule, early HCC, and HCC grade I-III. The boxplot of GSE89377 data demonstrates that the mRNA expression levels notably changed between low- and high-grade dysplastic nodules during carcinogenesis (Figure 4C). In the TCGA dataset, TRPV6 mRNA expression was lower in HCC tissue than in non-tumor liver tissue $(p<0.001$, Figure $4 \mathrm{E})$, and the group with low TRPV6 mRNA expression in the TCGA HCC cohort showed shorter progression-free survival than the high expression group ( $p=0.019$, Figure $4 \mathrm{~F})$, but not a significant difference in DSS ( $p=0.81$, Figure $4 \mathrm{G})$. 

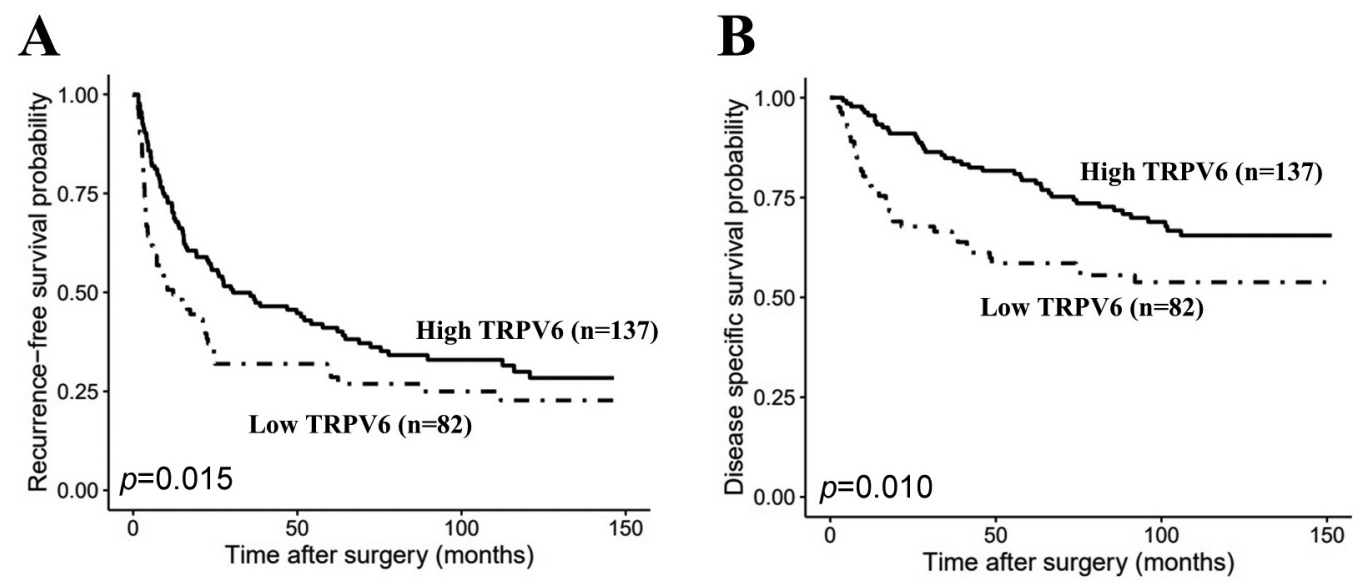

Figure 3. Kaplan-Meier survival curves for (A) RFS and (B) DSS according to TRPV6 H-score in our cohort. RFS: Recurrence-free survival; DSS: disease-specific survival.

\section{Discussion}

In this study, we found that low TRPV6 expression predicted adverse prognosis after curative $\mathrm{HCC}$ resection and was closely associated with aggressive clinicopathological parameters, such as large tumor size, high Edmonson grade, microvascular invasion, major portal vein invasion, tumor necrosis, intrahepatic metastasis, advanced AJCC or BCLC stage, and high serum AFP. We also found a lower expression of TRPV6 mRNA in HCC tissue than in non-tumor liver tissue in the various public datasets and confirmed its adverse prognostic value for progression-free survival in the TCGA dataset. These results suggest that down-regulation of TRPV6 could contribute to HCC progression.

TRPV6, an epithelial $\mathrm{Ca}^{2+}$ channel, has received attention because of its potential role in carcinogenesis due to its high $\mathrm{Ca}^{2+}$ selectivity. $\mathrm{Ca}^{2+}$ is a key messenger that regulates many signaling pathways involved in cellular processes, and the calcium signaling machinery is known to be widely involved in the control of cell proliferation and apoptosis, and accounts for a large part of the hallmark processes of cancer during progression $(10,30,31)$. The role of TRPV6 in cancer progression needs to be further elucidated, but inducing the accumulation of intracellular calcium might promote tumor cell survival. Briefly, elevated cytosolic calcium can bind to calmodulin, which activates calcineurin, and subsequently dephosphorylates the nuclear factor of activated T-cells (NFAT) transcription factor, becoming the active form. NFAT is known to mediate the transcription of some genes involved in cell proliferation and migration $(31,32)$.

It has been reported that TRPV6 is up-regulated in tissue samples of several human malignancies, including prostate, breast, thyroid, colon, and ovarian carcinomas, relative to its expression in normal tissues $(8,33,34)$. Wissenbach et al. first suggested that TRPV6 mRNA may be up-regulated in prostate cancer (34), and later studies demonstrated that TRPV6 mRNA expression increased with Gleason score and the degree of metastasis $(35,36)$. In breast cancer tissue, TRPV6 mRNA was also up-regulated, and it can be regulated by hormones such as estrogen and progesterone. Likewise, tamoxifen down-regulates TRPV6 mRNA expression and directly inhibits calcium channel activity, suggesting that TRPV6 is involved in the anti-proliferative activity of tamoxifen. As the pharmacologic activity of tamoxifen was enhanced in the TRPV6-silenced state, TRPV6 could be a promising therapeutic target for estrogen or progesterone receptor-negative breast cancer (37). Moreover, in colon cancer, it was demonstrated that over-expression of TRPV6 was related to early-stage disease, and inhibition of TRPV6 expression led to the suppression of growth and induction of apoptosis in tumor cells (38). However, down-regulation of TRPV6 has been also reported in renal, esophageal, and nonsmall cell lung cancer (14-16). Wu et al. also found that vitamin $D$ receptor mRNA expression is significantly associated with that of TRPV6 in renal cell carcinoma, suggesting that altered vitamin $D$ receptor expression is associated with carcinogenesis via TRPV6 (16). Furthermore, TRPV6 was down-regulated in patients with esophageal squamous cell carcinoma in a study by Zhang et al. (14). Interestingly, stratified survival analysis showed opposite results according to sex; decreased expression correlated with unfavorable DSS in male patients and favorable DSS in female patients (14). Collectively, the overexpression of TRPV6 in cancers suggests that it might be a probable oncogene, while decreased TRPV6 expression in some cancers and the association between decreased expression and adverse outcomes suggest that it might also act as a tumor suppressor. Although the pathologic mechanism of 
GSE14520
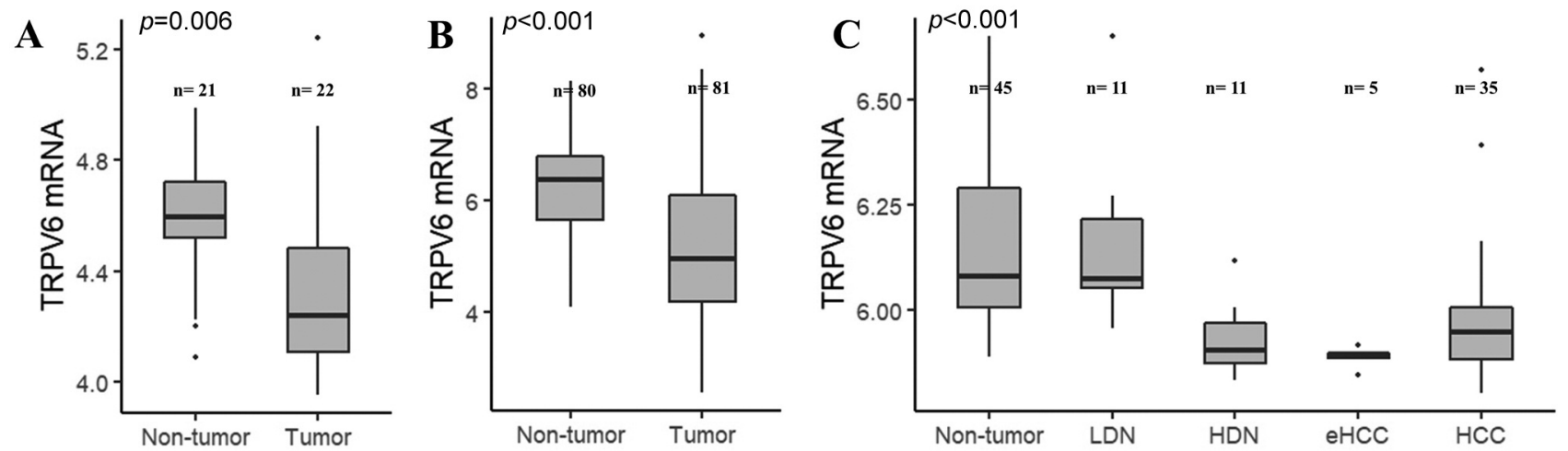

GSE149614

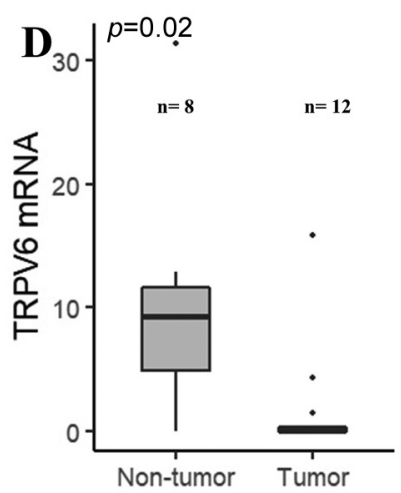

TCGA
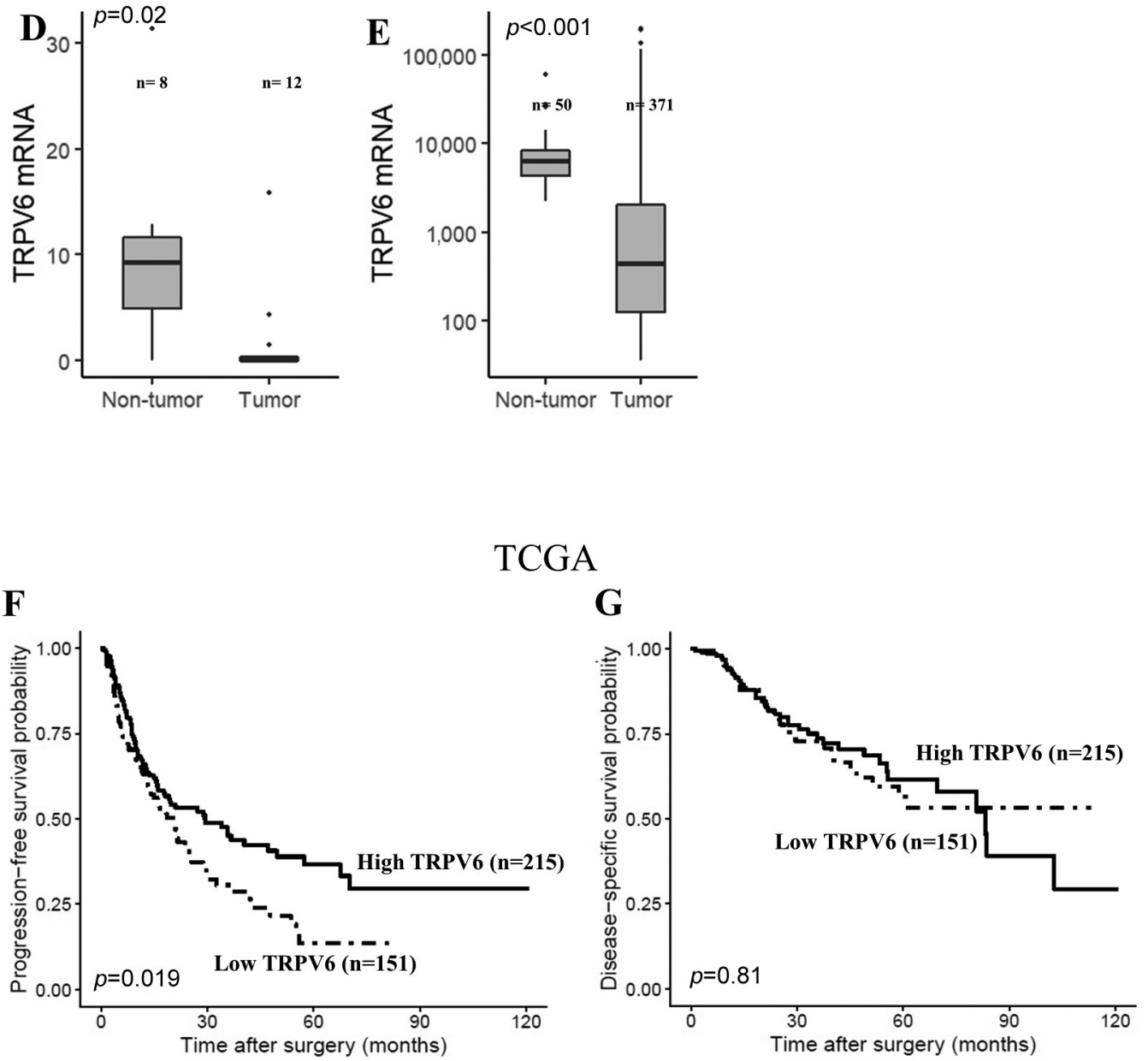

Figure 4. TRPV6 mRNA expression in public datasets. (A-E) Boxplot comparing TRPV6 mRNA expression between non-tumor tissue and HCC tumor tissue from public data. (A) In GSE14520, (B) in GSE54236, (C) in GSE 89377 (specifically, graded as non-tumor liver, LDN, HDN, early HCC, progressed HCC), (D) in GSE146914, and (E) in TCGA data. (F-G) Kaplan-Meier survival curves for PFS and DSS according to TRPV6 mRNA expression in TCGA cohort. LDN: Low-grade dysplastic nodule; HDN: high-grade dysplastic nodule; HCC: hepatocellular carcinoma; TCGA: The Cancer Genome Atlas; PFS: progression-free survival; DSS: disease-specific survival. 
TRPV6 in cancer tissue is not fully understood, it might play opposite and complicated roles in different cancer types; hence, further studies with large cohorts in various cancer types are required.

The association between TRPV6 expression at the protein or mRNA level and clinicopathological features of HCC have not yet been studied. However, previous studies have analyzed the clinical significance of TRPV1 and TRPV2 expression in patients with HCC. The expression of TRPV1 and TRPV2 at both mRNA and protein levels was lower in cancer tissue than in normal tissue, and it was also more likely in poorly-differentiated HCC than in welldifferentiated HCC. In particular, the high TRPV1 expression group had significantly better DFS than the low expression group $(39,40)$. These findings suggest that TRPV1 and TRPV2 play an important role in HCC carcinogenesis and could be potentially used as prognostic predictors of HCC.

Although HCC is one of the most common causes of cancer-related deaths worldwide, there is a lack of diversity in treatment, particularly for recurrent disease; therefore, new target agents are required for the treatment of HCC. Since recent studies asserting the role of TRP channels in cancer progression have emerged, efforts have been made to develop agents targeting TRP channels. Anand et al. reported that the venom peptide Tv1 from predatory marine snails has anticancer and anti-tumorigenic properties in vitro in liver cancer cells and in vivo in allograft tumor mouse models. They suggested that its mechanism is the inhibition of the COX-2 pathway via the NFAT signaling pathway following TRPV6 channel activity (41). In addition, inhibitory TRPV6binding peptides (SOR-C13 and SOR-C27) derived from soricidin, a paralytic peptide from the saliva of the northern short-tailed shrew, have been developed, and their antitumor activity has been proven in preclinical animal studies (42, 43). In a phase I clinical trial testing the safety and tolerability of SOR-C13 in 23 patients with advanced solid tumors of epithelial origin, the results of the "first-in-human" study revealed no drug-related adverse events, and the best response of $27 \%$ reduction in pancreatic tumor volume was observed (17). However, the application of these therapeutic agents against TRPV6 could be limited in HCC based on our study using real-world patient samples and clinical data, which showed weak expression of TRPV6 protein in nontumor liver tissue, the favorable prognostic effect of high TRPV6 expression, and lower mRNA expression of TRPV6 in the tumor than in the non-tumor liver.

\section{Conclusion}

To the best of our knowledge, this is the first study to investigate TRPV6 expression in HCC and its association with clinical outcomes. TRPV6 might be used as a prognostic biomarker in surgically resected HCC.

\section{Conflicts of Interest}

None of the authors have any conflicts of interest to declare regarding this study.

\section{Authors' Contributions}

Conception and design: SYH; Acquisition of data: HHK, SC, CP, SYH; Analysis and interpretation of data: HHK, SYH; Drafting the article: HHK, SYH; Revising and final approval of the article to be published: HHK, SYH; All Authors read and approved the final manuscript.

\section{Acknowledgements}

This research was supported by a grant from the Korea Health Technology R\&D Project through the Korea Health Industry Development Institute (KHIDI), funded by the Ministry of Health \& Welfare, Republic of Korea (grant number: HR20C0025), and an intramural grant from Samsung Medical Center (grant number: SMO1210411). The results published here are in part based on data generated by the TCGA Research Network: https://www.cancer.gov/ tcga.

\section{References}

1 Bray F, Ferlay J, Soerjomataram I, Siegel RL, Torre LA and Jemal A: Global cancer statistics 2018: GLOBOCAN estimates of incidence and mortality worldwide for 36 cancers in 185 countries. CA Cancer J Clin 68(6): 394-424, 2018. PMID: 30207593. DOI: $10.3322 /$ caac. 21492

2 Sherman M: Recurrence of hepatocellular carcinoma. N Engl J Med 359(19): 2045-2047, 2008. PMID: 18923166. DOI: 10.1056/NEJMe0807581

3 Bruix J, Qin S, Merle P, Granito A, Huang YH, Bodoky G, Pracht M, Yokosuka O, Rosmorduc O, Breder V, Gerolami R, Masi G, Ross PJ, Song T, Bronowicki JP, Ollivier-Hourmand I, Kudo M, Cheng AL, Llovet JM, Finn RS, LeBerre MA, Baumhauer A, Meinhardt G, Han G and RESORCE Investigators: Regorafenib for patients with hepatocellular carcinoma who progressed on sorafenib treatment (RESORCE): a randomised, double-blind, placebo-controlled, phase 3 trial. Lancet 389(10064): 56-66, 2017. PMID: 27932229. DOI: 10.1016/S0140-6736(16)32453-9

4 El-Khoueiry AB, Sangro B, Yau T, Crocenzi TS, Kudo M, Hsu C, Kim TY, Choo SP, Trojan J, Welling TH Rd, Meyer T, Kang YK, Yeo W, Chopra A, Anderson J, Dela Cruz C, Lang L, Neely J, Tang H, Dastani HB and Melero I: Nivolumab in patients with advanced hepatocellular carcinoma (CheckMate 040): an openlabel, non-comparative, phase $1 / 2$ dose escalation and expansion trial. Lancet 389(10088): 2492-2502, 2017. PMID: 28434648. DOI: 10.1016/S0140-6736(17)31046-2

5 Kudo M, Finn RS, Qin S, Han KH, Ikeda K, Piscaglia F, Baron A, Park JW, Han G, Jassem J, Blanc JF, Vogel A, Komov D, Evans TRJ, Lopez C, Dutcus C, Guo M, Saito K, Kraljevic S, Tamai T, Ren M and Cheng AL: Lenvatinib versus sorafenib in first-line treatment of patients with unresectable hepatocellular carcinoma: a randomised phase 3 non-inferiority trial. Lancet 391(10126): 1163-1173, 2018. PMID: 29433850. DOI: $10.1016 / \mathrm{S} 0140-6736(18) 30207-1$ 
6 Zhu AX, Finn RS, Edeline J, Cattan S, Ogasawara S, Palmer D, Verslype C, Zagonel V, Fartoux L, Vogel A, Sarker D, Verset G, Chan SL, Knox J, Daniele B, Webber AL, Ebbinghaus SW, Ma $\mathrm{J}$, Siegel AB, Cheng AL, Kudo $\mathrm{M}$ and KEYNOTE-224 investigators: Pembrolizumab in patients with advanced hepatocellular carcinoma previously treated with sorafenib (KEYNOTE-224): a non-randomised, open-label phase 2 trial. Lancet Oncol 19(7): 940-952, 2018. PMID: 29875066. DOI: 10.1016/S1470-2045(18)30351-6

7 Qin LX and Tang ZY: Recent progress in predictive biomarkers for metastatic recurrence of human hepatocellular carcinoma: a review of the literature. J Cancer Res Clin Oncol 130(9): 497-513, 2004. PMID: 15205947. DOI: 10.1007/s00432-0040572-9

8 Lehen'kyi V, Raphaël M and Prevarskaya N: The role of the TRPV6 channel in cancer. J Physiol 590(6): 1369-1376, 2012. PMID: 22331416. DOI: 10.1113/jphysiol.2011.225862

9 den Dekker E, Hoenderop JG, Nilius B and Bindels RJ: The epithelial calcium channels, TRPV5 \& TRPV6: from identification towards regulation. Cell Calcium 33(5-6): 497-507, 2003. PMID: 12765695. DOI: 10.1016/s0143-4160(03)00065-4

10 Bruce JIE and James AD: Targeting the calcium signalling machinery in cancer. Cancers (Basel) 12(9): 2351, 2020. PMID: 32825277. DOI: $10.3390 /$ cancers 12092351

11 Martinac B: The ion channels to cytoskeleton connection as potential mechanism of mechanosensitivity. Biochim Biophys Acta 1838(2): 682-691, 2014. PMID: 23886913. DOI: 10.1016/j.bbamem.2013.07.015

12 Wissenbach U, Niemeyer B, Himmerkus N, Fixemer T, Bonkhoff $\mathrm{H}$ and Flockerzi V: TRPV6 and prostate cancer: cancer growth beyond the prostate correlates with increased TRPV6 Ca2+ channel expression. Biochem Biophys Res Commun 322(4): 13591363, 2004. PMID: 15336984. DOI: 10.1016/j.bbrc.2004.08.042

13 Ouadid-Ahidouch H, Dhennin-Duthille I, Gautier M, Sevestre H and Ahidouch A: TRP calcium channel and breast cancer: expression, role and correlation with clinical parameters. Bull Cancer 99(6): 655-664, 2012. PMID: 22640890. DOI: 10.1684/ bdc. 2012.1595

14 Zhang SS, Xie X, Wen J, Luo KJ, Liu QW, Yang H, Hu Y and $\mathrm{Fu}$ JH: TRPV6 plays a new role in predicting survival of patients with esophageal squamous cell carcinoma. Diagn Pathol 11: 14, 2016. PMID: 26818094. DOI: 10.1186/s13000-016-0457-7

15 Fan H, Shen YX and Yuan YF: Expression and prognostic roles of TRPV5 and TRPV6 in non-small cell lung cancer after curative resection. Asian Pac J Cancer Prev 15(6): 2559-2563, 2014. PMID: 24761864. DOI: 10.7314/apjcp.2014.15.6.2559

$16 \mathrm{Wu}$ Y, Miyamoto T, Li K, Nakagomi H, Sawada N, Kira S, Kobayashi H, Zakohji H, Tsuchida T, Fukazawa M, Araki I and Takeda M: Decreased expression of the epithelial $\mathrm{Ca}^{2+}$ channel TRPV5 and TRPV6 in human renal cell carcinoma associated with vitamin D receptor. J Urol 186(6): 2419-2425, 2011. PMID: 22019165. DOI: $10.1016 /$ j.juro.2011.07.086

17 Fu S, Hirte H, Welch S, Ilenchuk TT, Lutes T, Rice C, Fields N, Nemet A, Dugourd D, Piha-Paul S, Subbiah V, Liu L, Gong J, Hong D and Stewart JM: First-in-human phase I study of SORC13, a TRPV6 calcium channel inhibitor, in patients with advanced solid tumors. Invest New Drugs 35(3): 324-333, 2017. PMID: 28150073. DOI: 10.1007/s10637-017-0438-Z

18 Amin MB, Edge SB, Greene F, Byrd DR, Brookland RK, Washington MK, Gershenwald JE, Compton CC, Hess KR,
Sullivan DC, Jessup JM, Brierley JD, Gaspar LE, Schilsky RL, Balch CM, Winchester DP, Asare EA, Madera M, Gress DM and Meyer LR: AJCC Cancer Staging Manual. 8th ed. New York, Springer, 2017.

19 Llovet JM, Brú C and Bruix J: Prognosis of hepatocellular carcinoma: the BCLC staging classification. Semin Liver Dis 19(3): 329-338, 1999. PMID: 10518312. DOI: 10.1055/s-2007-1007122

20 Kumada T, Nakano S, Takeda I, Sugiyama K, Osada T, Kiriyama S, Sone Y, Toyoda H, Shimada S, Takahashi M and Sassa T: Patterns of recurrence after initial treatment in patients with small hepatocellular carcinoma. Hepatology 25(1): 87-92, 1997. PMID: 8985270. DOI: 10.1053/jhep.1997.v25.pm0008985270

21 Ha SY, Choi S, Park S, Kim JM, Choi GS, Joh JW and Park CK: Prognostic effect of preoperative neutrophil-lymphocyte ratio is related with tumor necrosis and tumor-infiltrating lymphocytes in hepatocellular carcinoma. Virchows Arch 477(6): 807-816, 2020. PMID: 32483662. DOI: 10.1007/s00428-020-02841-5

22 Ha SY, Kim JH, Yang JW, Kim J, Kim B and Park CK: The overexpression of CCAR1 in hepatocellular carcinoma associates with poor prognosis. Cancer Res Treat 48(3): 10651073, 2016. PMID: 26511806. DOI: 10.4143/crt.2015.302

23 Lee T, Park CK and Ha SY: Prognostic role of apelin receptor expression in hepatocellular carcinoma treated with curative surgical resection. Anticancer Res 39(6): 3025-3031, 2019. PMID: 31177144. DOI: 10.21873/anticanres.13435

24 Roessler S, Jia HL, Budhu A, Forgues M, Ye QH, Lee JS, Thorgeirsson SS, Sun Z, Tang ZY, Qin LX and Wang XW: A unique metastasis gene signature enables prediction of tumor relapse in early-stage hepatocellular carcinoma patients. Cancer Res 70(24): 10202-10212, 2010. PMID: 21159642. DOI: 10.1158/0008-5472.CAN-10-2607

25 Villa E, Critelli R, Lei B, Marzocchi G, Cammà C, Giannelli G, Pontisso P, Cabibbo G, Enea M, Colopi S, Caporali C, Pollicino T, Milosa F, Karampatou A, Todesca P, Bertolini E, Maccio L, Martinez-Chantar ML, Turola E, Del Buono M, De Maria N, Ballestri S, Schepis F, Loria P, Enrico Gerunda G, Losi L and Cillo U: Neoangiogenesis-related genes are hallmarks of fastgrowing hepatocellular carcinomas and worst survival. Results from a prospective study. Gut 65(5): 861-869, 2016. PMID: 25666192. DOI: 10.1136/gutjnl-2014-308483

26 Shen Q, Eun JW, Lee K, Kim HS, Yang HD, Kim SY, Lee EK, Kim T, Kang K, Kim S, Min DH, Oh SN, Lee YJ, Moon H, Ro SW, Park WS, Lee JY and Nam SW: Barrier to autointegration factor 1, procollagen-lysine, 2-oxoglutarate 5-dioxygenase 3, and splicing factor $3 \mathrm{~b}$ subunit 4 as early-stage cancer decision markers and drivers of hepatocellular carcinoma. Hepatology 67(4): 13601377, 2018. PMID: 29059470. DOI: 10.1002/hep.29606

27 Li C, Chen J, Li Y, Wu B, Ye Z, Tian X, Wei Y, Hao Z, Pan Y, Zhou $\mathrm{H}$, Yang $\mathrm{K}, \mathrm{Fu} \mathrm{Z}, \mathrm{Xu} \mathrm{J}$ and $\mathrm{Lu} \mathrm{Y}$ : 6phosphogluconolactonase promotes hepatocellular carcinogenesis by activating pentose phosphate pathway. Front Cell Dev Biol 9: 753196, 2021. PMID: 34765603. DOI: 10.3389/fcell.2021.753196

28 Cancer Genome Atlas Research Network: Comprehensive and integrative genomic characterization of hepatocellular carcinoma. Cell 169(7): 1327-1341.e23, 2017. PMID: 28622513. DOI: $10.1016 /$ j.cell.2017.05.046

29 Camp RL, Dolled-Filhart M and Rimm DL: X-tile: a new bioinformatics tool for biomarker assessment and outcome-based cut-point optimization. Clin Cancer Res 10(21): 7252-7259, 2004. PMID: 15534099. DOI: 10.1158/1078-0432.CCR-04-0713 
30 Santoni G, Farfariello V and Amantini C: TRPV channels in tumor growth and progression. Adv Exp Med Biol 704: 947-967, 2011. PMID: 21290335. DOI: 10.1007/978-94-007-0265-3 49

31 Huber SM: Oncochannels. Cell Calcium 53(4): 241-255, 2013. PMID: 23357407. DOI: 10.1016/j.ceca.2013.01.001

32 Crabtree GR and Olson EN: NFAT signaling: choreographing the social lives of cells. Cell 109 Suppl: S67-S79, 2002. PMID: 11983154. DOI: 10.1016/s0092-8674(02)00699-2

33 Zhuang L, Peng JB, Tou L, Takanaga H, Adam RM, Hediger MA and Freeman MR: Calcium-selective ion channel, CaT1, is apically localized in gastrointestinal tract epithelia and is aberrantly expressed in human malignancies. Lab Invest 82(12): 1755-1764, 2002. PMID: 12480925. DOI: 10.1097/01.lab.0000043910.41414.e7

34 Wissenbach U, Niemeyer BA, Fixemer T, Schneidewind A, Trost C, Cavalie A, Reus K, Meese E, Bonkhoff H and Flockerzi V: Expression of CaT-like, a novel calcium-selective channel, correlates with the malignancy of prostate cancer. J Biol Chem 276(22): 19461-19468, 2001. PMID: 11278579. DOI: $10.1074 / \mathrm{jbc} . \mathrm{M} 009895200$

35 Fixemer $T$, Wissenbach $U$, Flockerzi $\mathrm{V}$ and Bonkhoff $\mathrm{H}$ : Expression of the $\mathrm{Ca}^{2+}$-selective cation channel TRPV6 in human prostate cancer: a novel prognostic marker for tumor progression. Oncogene 22(49): 7858-7861, 2003. PMID: 14586412. DOI: $10.1038 /$ sj.onc.1206895

36 Peng JB, Zhuang L, Berger UV, Adam RM, Williams BJ, Brown EM, Hediger MA and Freeman MR: CaT1 expression correlates with tumor grade in prostate cancer. Biochem Biophys Res Commun 282(3): 729-734, 2001. PMID: 11401523. DOI: 10.1006/bbrc. 2001.4638

37 Bolanz KA, Hediger MA and Landowski CP: The role of TRPV6 in breast carcinogenesis. Mol Cancer Ther 7(2): 271279, 2008. PMID: 18245667. DOI: 10.1158/1535-7163.MCT-070478

38 Peleg S, Sellin JH, Wang Y, Freeman MR and Umar S: Suppression of aberrant transient receptor potential cation channel, subfamily $\mathrm{V}$, member 6 expression in hyperproliferative colonic crypts by dietary calcium. Am J Physiol Gastrointest Liver Physiol 299(3): G593-G601, 2010. PMID: 20508153 DOI: 10.1152/ajpgi.00193.2010
39 Miao X, Liu G, Xu X, Xie C, Sun F, Yang Y, Zhang T, Hua S, Fan W, Li Q, Huang S, Wang Q, Liu G and Zhong D: High expression of vanilloid receptor-1 is associated with better prognosis of patients with hepatocellular carcinoma. Cancer Genet Cytogenet 186(1): 25-32, 2008. PMID: 18786439. DOI: 10.1016/j.cancergencyto.2008.05.011

40 Liu G, Xie C, Sun F, Xu X, Yang Y, Zhang T, Deng Y, Wang D, Huang Z, Yang L, Huang S, Wang Q, Liu G, Zhong D and Miao $\mathrm{X}$ : Clinical significance of transient receptor potential vanilloid 2 expression in human hepatocellular carcinoma. Cancer Genet Cytogenet 197(1): 54-59, 2010. PMID: 20113837. DOI: 10.1016/j.cancergencyto.2009.08.007

41 Anand P, Filipenko P, Huaman J, Lyudmer M, Hossain M, Santamaria C, Huang K, Ogunwobi OO and Holford M: Antitumor effects of Tv1 venom peptide in liver cancer. bioRxiv: 518340, 2019. DOI: 10.1101/518340

42 Bowen CV, DeBay D, Ewart HS, Gallant P, Gormley S, Ilenchuk TT, Iqbal U, Lutes T, Martina M, Mealing G, Merkley N, Sperker S, Moreno MJ, Rice C, Syvitski RT and Stewart JM: In vivo detection of human TRPV6-rich tumors with anti-cancer peptides derived from soricidin. PLoS One 8(3): e58866, 2013. PMID: 23554944. DOI: 10.1371/journal.pone.0058866

43 Xue H, Wang Y, MacCormack TJ, Lutes T, Rice C, Davey M, Dugourd D, Ilenchuk TT and Stewart JM: Inhibition of Transient Receptor Potential Vanilloid 6 channel, elevated in human ovarian cancers, reduces tumour growth in a xenograft model. J Cancer 9(17): 3196-3207, 2018. PMID: 30210643. DOI: $10.7150 /$ jca.20639 